Check for updates

Cite this: RSC Adv., 2019, 9, 13615

Received 19th February 2019

Accepted 8th April 2019

DOI: 10.1039/c9ra01267b

rsc.li/rsc-advances

\section{Exfoliated fluorinated carbons with a low and stable friction coefficient $\uparrow$}

\author{
Michael Herraiz, ${ }^{a}$ Marc Dubois, (D) ${ }^{* a}$ Nicolas Batisse, ${ }^{a}$ Elodie Petit ${ }^{a}$ \\ and Philippe Thomas ${ }^{b}$
}

Exfoliation appears as a promising way to decrease the friction coefficient of carbon materials. Although there is massive defluorination during exfoliation, the friction coefficient is not increased and an exfoliated structure facilitates the formation of a homogeneous and stable tribofilm. The weakening of the interparticle interactions due to the exfoliation process is the main explanation for the excellent tribological properties. Three representative examples are studied to evidence the efficiency of the thermal shock to prepare solid lubricants or additives for lubricating oils, high temperature graphite fluorides, fluorinated carbon nanofibers and fluorinated nanodiscs. An opened (graphite fluoride) or defect structure (nanofibers) allows the gases formed during the exfoliation to be evolved; the exfoliation is then successful regardless of the $\mathrm{C}-\mathrm{F}$ bonding. Exfoliation and defluorination occur simultaneously resulting in samples with a low F/C atomic ratio. On the contrary for the case of fluorinated nanodiscs, the exfoliation fails because of cracks and edges as well as the low diameter of the discs.

\section{Introduction}

Few layer graphene is found to have exceptional anti-friction properties. ${ }^{\mathbf{1 - 4}}$ The addition of graphene material in lubricating oil or fluid, even in a small fraction, results in a significant decrease of wear and the friction coefficient. The surface chemistry of graphene can be tailored either during the synthesis or by posttreatment. Addition of oxygenated, hydrogenated or fluorinated groups results in opposite effects on friction properties. Regarding oxidized graphene, ${ }^{5}$ the friction between two sliding graphene layers increases with the introduction of oxygenated groups; epoxide and hydroxyl groups increase electrostatic and form nonstable hydrogen bond interactions. A higher energy barrier must be overcome resulting in high energy dissipation. On the contrary, with hydrogenated graphene sheets, the friction decreases in comparison with pure graphene. ${ }^{6}$ Electrons are accumulated between the carbon atoms and the attached hydrogen atoms, resulting in the repulsion of two interacting sheets and thus in decreased friction energy. Because of the higher electronegativity of fluorine, such an effect is expected to be much higher when those atoms are present instead of hydrogen. ${ }^{7}$ For fluorinated 0D, 1D and 2D nanocarbons, namely graphitized carbon blacks, nanofibers and nanodiscs, which exhibit similar covalent CF bonding and a closed structure, good tribological properties were

${ }^{a}$ Université Clermont Auvergne, CNRS, SIGMA Clermont, Institut de Chimie de Clermont-Ferrand, F-63000 Clermont-Ferrand, France.E-mail: marc.dubois@uca.fr ${ }^{b}$ Groupe de Technologie des Surfaces et Interfaces, Université des Antilles Campus Fouillole, BP250 97157 Pointe à Pitre Cedex, France

$\dagger$ Electronic supplementary information (ESI) available. See DOI: 10.1039/c9ra01267b achieved whatever the fluorine content and those nanomaterials appear as excellent precursors for the tribo-active phase in lubrication. ${ }^{8-11}$ Indeed the friction coefficients of the fluorinated nanocarbons ranged between 0.065 and 0.085 , and are weaker than those of most solid lubricants (graphite). Accommodation of fluorine atoms into the carbon lattice with a $\mathrm{F} / \mathrm{C}$ atomic ratio higher than 0.15 results in easy cleavage for the cases of fluorinated carbon blacks and nanofibers. ${ }^{7-11}$

The aim of the present paper is to investigate the tribological properties of graphene materials, which are prepared by an easy operating, highly scalable, and low-cost method by applying a fast thermal treatment on highly fluorinated carbons under argon atmosphere. This route differs from the three conventional synthesis methods to obtain fluorinated graphene; all require mild conditions as follows: (i) reaction between raw graphene and fluorinating agent such as $\mathrm{XeF}_{2}$ or $\mathrm{F}_{2}$, (ii) exfoliation of (highly) fluorinated graphite, (iii) fluorination of graphene oxide (GO). In order to evaluate the efficiency for different fluorinated carbons, three precursors were studied, graphite fluoride with $\mathrm{CF}_{1}$ composition as a reference compound, 1D fluorinated nanofibers (CNF) and 2D fluorinated nanodiscs (CND). The aim is to highlight the effect of the morphology.

\section{Experimental}

\subsection{Starting materials}

Fluorination of commercial KS15 graphite (TIMCAL) with 15 $\mu \mathrm{m}$ grain size was carried out with undiluted gaseous $\mathrm{F}_{2}$ at $1 \mathrm{~atm}$ pressure under dynamic condition in a flow reactor. Fluorination at high temperature and for long exposition period, in our 
case $600{ }^{\circ} \mathrm{C}$ for 6 hours, results in covalent fluorinated graphite with $(\mathrm{CF})_{n}$ structural type and $\mathrm{CF}_{1}$ chemical composition. The resulting graphite fluoride is denoted as $F G$. After fluorination, the chemical composition, estimated by Nuclear Magnetic Resonance (NMR) was $\mathrm{CF}_{1}$.

High-purity (>90\%) CNF, 2-20 $\mu \mathrm{m}$ in length (supplied by courtesy of the MER Corporation, Tucson, Arizona) were obtained by chemical vapor deposition (CVP) and heat treated at $1800{ }^{\circ} \mathrm{C}$ in an argon atmosphere to enhance their graphitization degree. Their diameter distribution is between 80 and $350 \mathrm{~nm}$. The average diameter is estimated equal to $150 \mathrm{~nm}$ from observations of various parts of the raw sample. The welldefined periodicity of the layers (interlayer distance $d$ of 0.337 $\mathrm{nm}$ ) was confirmed both by transmission electron microscopy and X-ray diffraction.

The 2D samples consist in a mixture of carbon nanodiscs (70 weight\%), carbon nanocones (20 weight\%) and amorphous carbon (10 weight\%), provided by NTec Norway annealed under argon at $2700{ }^{\circ} \mathrm{C}$ for graphitization. ${ }^{12}$ Because of the large amount of discs, the mixture will be denoted CNDs. CNDs were produced by pyrolysis of heavy oil using the Kvaerner Carbon Black and Hydrogen Process $(\mathrm{CBH}) .{ }^{13}$

Direct fluorination was carried out with pure fluorine gas flow in a Monel reactor. Different fluorine contents were obtained according to the fluorination temperatures $T_{\mathrm{F}}$ with constant duration of $3 \mathrm{~h}$ and $\mathrm{F}_{2}$ gas flux of $10 \mathrm{~mL} \mathrm{~min}{ }^{-1}$. The fluorine content " $x$ " of the fluorinated nanocarbons, expressed as the atomic $\mathrm{F} / \mathrm{C}$ ratio, was determined first by gravimetry upon fluorination (weight uptake method) and confirmed by quantitative ${ }^{19} \mathrm{~F}$ NMR analyses. Carbon nanofibers and nanodiscs were placed treated at temperatures $T_{\mathrm{F}}$ equal to 480 and $520{ }^{\circ} \mathrm{C}$, respectively. The resulting materials, denoted as FCNF and FCND, exhibit both $x$ value of $0.80 \pm 0.05$.

\subsection{Exfoliation}

The exfoliation of the starting materials was carried out by a fast thermal shock induced by a system composed of a metallic resistance wrapped around a closed silica reactor and connected to an electric power supply. In order to avoid oxidation during the exfoliation, the experiment was performed under argon atmosphere. The fast increase in temperature (around $10{ }^{\circ} \mathrm{C} \mathrm{s}^{-1}$ ) causes decomposition of the sample, inducing the generation of fluorinated gaseous species in a very short time and as the result, the exfoliation of the material. Moreover, during this fast step, temperature was measured to be invariant for $F G$ and $F C N F$ because the energy released by the flame was consumed by the medium. FCND exhibited a different behavior with a continuous temperature increase. Whatever the starting material FCND, both the volume expansion and color change from white to black (see Fig. SI1 in ESI $\dagger$ ). The final treatment temperatures, which were measured on the samples thanks to a thermocouple temperature sensor were 682 , and $584{ }^{\circ} \mathrm{C}$ for $F G$ and $F \mathrm{CNF}$, respectively. Such a values are in good accordance with the fluorination temperature of 600 and $480{ }^{\circ} \mathrm{C}$ to prepare the precursor. The higher the fluorination temperature, the higher the thermal stability. For the exfoliated samples are called Exf-FG, Exf-FCNF and Exf-FCND.

\subsection{Tribological tests}

The tribologic properties of the compounds were evaluated under air atmosphere (relative humidity: $50 \pm 5 \%$ ) using a ballon-plane tribometer in which a ball describes a reciprocating motion on a static steel plane. The tested material was deposited onto a static AISI 52100 steel plane. In our experimental conditions, one friction cycle corresponds to one second. The tribologic experiments duration was 1000 seconds (1000 cycles of friction).

Prior to friction tests, the planes were polished to generate multidirectional scratches and to obtain an average peak-topeak roughness of $350 \mathrm{~nm}$ in order to improve the adherence of the tested materials. The balls were used as delivered (average peak-to-peak roughness of $90 \mathrm{~nm}$ ). The balls and the planes were cleaned in ultrasonic baths in acetone and ethanol in order to eliminate pollutants and remaining abrasive particles. Then few milligrams of powdery carbon phases were deposited on the plane surface. A normal load of $10 \mathrm{~N}$ was applied. The ball moved on the static plane surface at a speed of $3 \mathrm{~mm} \mathrm{~s}^{-1}$. According th Hertz's theory, the contact diameter was $140 \mu \mathrm{m}$ and the mean contact pressure was $0.65 \mathrm{GPa}$. The friction coefficient $\mu$ was measured with a computer-based data acquisition system. The tribological parameters $\left(10 \mathrm{~N}, 3 \mathrm{~mm} \mathrm{~s}^{-1}\right)$ were selected in order to compare with other fluorinated (nano) carbons. The experimental conditions are chosen in order to test the tribological performances of the compounds in the boundary lubrication regime.

\subsection{Characterization}

Scanning electron microscopy (SEM) micrographs were recorded using a Zeiss SUPRA 55VP SEM operating at $3 \mathrm{kV}$.

Fourier Transform Infrared spectroscopy (FTIR) was carried out on solid products using a Thermo Nicolet 5700 in Attenuated Total Reflectance (ATR) configuration.

Raman spectra were recorded at room temperature using a Jobin Yvon T64000 with a charge coupled device multichannel detector. The radiation source was a $514.5 \mathrm{~nm}$ Argon laser line. The laser power was tuned to $10 \mathrm{~mW}$.

NMR experiments were carried out with Bruker Avance spectrometer, with working frequencies for ${ }^{13} \mathrm{C}$ and ${ }^{19} \mathrm{~F}$ of 73.4 and 282.2 $\mathrm{MHz}$, respectively. A Magic Angle Spinning (MAS) probe (Bruker) operating with $2.5 \mathrm{~mm}$ rotors was used. For MAS spectra, a simple sequence was performed with a single $\pi / 2$ pulse length of 4.0 and $3.5 \mu$ s for ${ }^{19} \mathrm{~F}$ and ${ }^{13} \mathrm{C}$, respectively. ${ }^{13} \mathrm{C}$ chemical shifts were externally referenced to tetramethylsilane (TMS). ${ }^{19} \mathrm{~F}$ chemical shifts were referenced with respect to $\mathrm{CFCl}_{3}$.

\section{Results and discussion}

\subsection{Materials}

The efficiency of the exfoliation may be highlighted by the morphology of the resulting samples (Fig. 1). SEM images of 
Exf-FG, shown in Fig. 1a and b, reveal an expanded architecture, typical for thermally exfoliated graphite. The fast generation of fluorinated gases during the flash allowed the porosity of the sample to be increased, and the specific surface to be developed. All particles appear exfoliated independently of their initial expansion. TEM confirms the change of morphology and formation of multilayer graphene (Fig. 2). The morphology of CNF is also drastically changed. The fibers appears opened along their longitudinal axe (Fig. 1c and d). In order to understand those changes, fluorination mechanism must be considered. The fluorination of those nanofibers started on the defective or unsaturated carbons sitting at outlayers or the nanofiber tips (although their amount is quite low since the sample were post-treated at $1800{ }^{\circ} \mathrm{C}$ under argon atmosphere). The fluorination could then progress toward the core through an opening of some external walls in order to permit the volume expansion resulting from the accommodation of fluorine atoms and the carbon hybridization change from $\mathrm{sp}^{2}$ into $\mathrm{sp}^{3}$ (see Fig. 3). Initially the nanofibers appears as a closed structure similar to multiwall carbon nanotubes MWCNTs; they become a quasi-opened structure with numerous defects, i.e. disrupted walls which allow the exfoliation to start and progress during the thermal. The walls are then expanded (Fig. 1c and d). On the contrary, most of the discs and cones are still present after the exfoliation (Fig. 1e and f). A swelling of both the discs and cones occurred but without exfoliation as for $G F$. Nanodiscs are expected as an opened structure which consists in the stacking of discs. The weak exfoliation is surprising and a careful investigation of the discs is necessary to understand this experimental fact.

After that the exfoliation was demonstrated for $F G$ and $F C N F$, the content of fluorine atoms was addressed using solid state NMR. ${ }^{19}$ F NMR experiments were performed using MAS with spinning speed of $34 \mathrm{kHz}$ (Fig. 4). With such conditions, the strong ${ }^{19} \mathrm{~F}-{ }^{19} \mathrm{~F}$ homonuclear dipolar coupling and possible chemical shift anisotropy are either averaged or decreased, that results in a substantial narrowing and separation of the lines.

Whatever the starting material, the low signal/noise ratio in the spectra of the exfoliated sample (although a high scan number of 3072 has been applied) reveals the low fluorine content. Concerning the covalence of C-F bonding, the lower the ${ }^{19} \mathrm{~F}$ chemical shift, the higher the covalence. ${ }^{14}$ The $\mathrm{C}-\mathrm{F}$ bonding for $F G, F C N F$ and FCND is mostly covalent as revealed by the single line at $-190 \mathrm{ppm}$, whereas for the exfoliated samples, both covalent $\mathrm{C}-\mathrm{F}$ bonds and $\mathrm{C}-\mathrm{F}$ with weakened covalence coexist; their lines overlap in the $-200 /-150 \mathrm{ppm}$ range. $\mathrm{CF}_{2}$ and $\mathrm{CF}_{3}$ are present in all raw and exfoliated samples as revealed by the lines at -120 and $-80 \mathrm{ppm}$, respectively. Whereas those groups are present in very few amounts for the starting materials, their relative contents in Exf-FG are respectively 60.4 and $23.4 \%$ regarding fluorine atoms (Table 1 ). Those data are extracted from the fits with Lorentzian lines (Fig. 4b). The relative content of CF bonds is $16.2 \%$ (initially $100 \%$ ) highlighting that a drastic defluorination occurs during the exfoliation. The data for exfoliated FCNF are close (6.8, 69.9 and $23.3 \%$ for $\mathrm{CF}, \mathrm{CF}_{2}$ and $\mathrm{CF}_{3}$, respectively) and similar exfoliation/ defluorination mechanism may occur. On the contrary, $80.8 \%$ of $\mathrm{CF}$ is found for only $2.3 \%$ of $\mathrm{CF}_{2}$ and $16.9 \%$ of $\mathrm{CF}_{3}$. $\mathrm{CF}$ groups are located along the sheets whereas $\mathrm{CF}_{2}$ and $\mathrm{CF}_{3}$ are at the sheet edges and/or at the defects. The slight differences in their contents depend of both the sheet size and disorder. The weak exfoliation revealed by SEM images, occurred without change in the $\mathrm{C}-\mathrm{F}$ bonding; the main group is still $\mathrm{CF}$.

For the cases of $F G$ and $F C N F$, thermal exfoliation results in sheet disruptions and sheet edges fix fluorine atoms in $\mathrm{CF}_{2}$ and $\mathrm{CF}_{3}$ groups. Thermal defluorination with $\mathrm{CF}_{4}$ and $\mathrm{C}_{2} \mathrm{~F}_{6}$ result in a local breaking of the graphene sheets that also is in favor of the exfoliation step. ${ }^{15}$ On the contrary, the amount of on-plane fluorine atoms decreases partly by relocation from the bulk to the defects/edges.

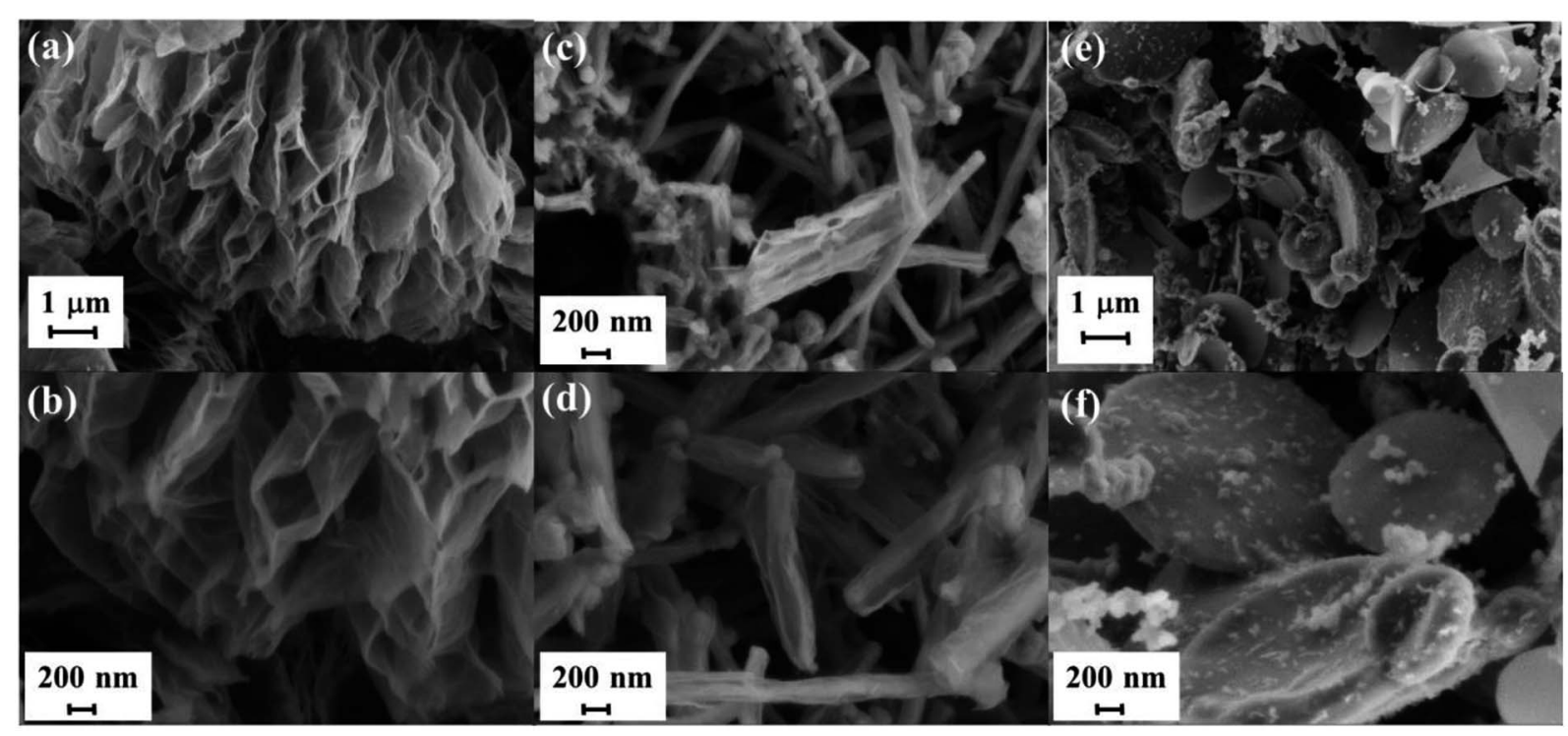

Fig. 1 SEM images of thermally exfoliated FG ( $a$ and b), FCNF (c and d) and FCND (e and f). 


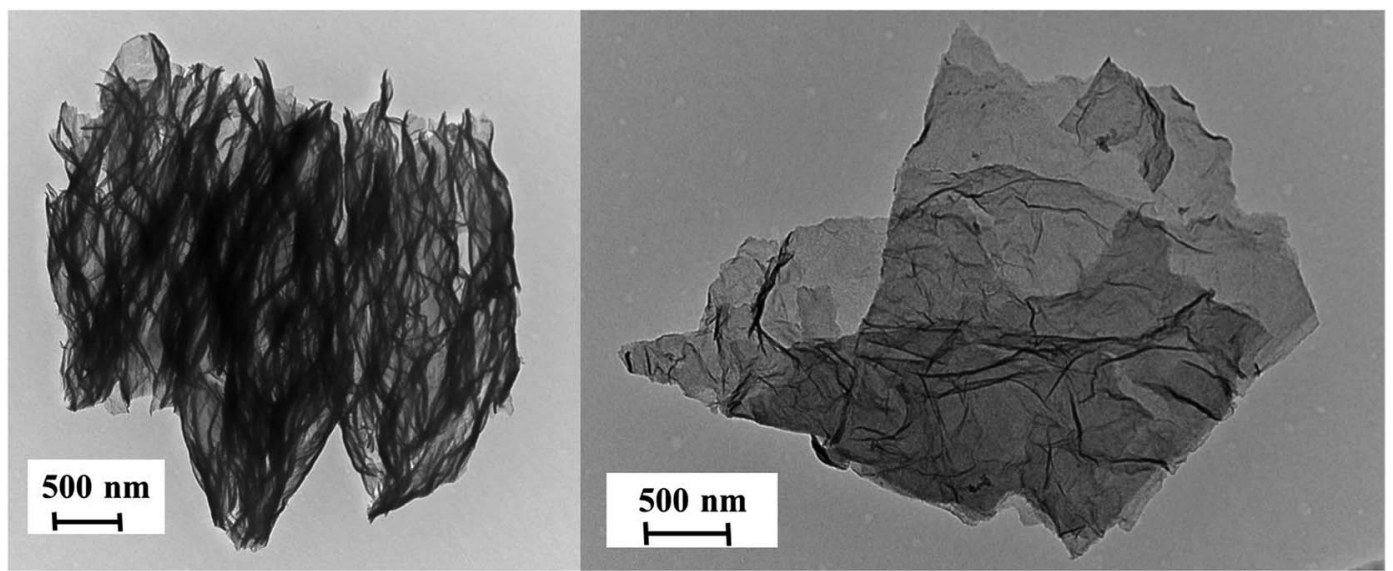

Fig. 2 TEM images of thermally exfoliated FG.

The fast generation of gas species favors the exfoliation. In addition, the initial presence of fluorine atoms between the layers decreases the van der Waals interactions and facilitates this process. By analogy with conventional thermal process, ${ }^{15}$ large-scale defluorination is achieved due to the temperature and the weakened covalence of the $\mathrm{C}-\mathrm{F}$ bonds. The lower the fluorine content, the higher the weakening of the $\mathrm{C}-\mathrm{F}$ bond covalence by hyperconjugation (between $\mathrm{C}-\mathrm{F}$ and nonfluorinated $\mathrm{sp}^{2}$ carbon atoms in the neighboring). ${ }^{16}$ The two steps of exfoliation/restructuration occur quite simultaneously during the flash thermal.

Defluorination during exfoliation is confirmed by infrared spectroscopy (Fig. 5a). For the fluorinated precursors, the main band at $1199 \mathrm{~cm}^{-1}$ is characteristic of the stretching vibration of C-F covalent bond, whereas the shoulder present near $1120 \mathrm{~cm}^{-1}$ is related to $\mathrm{C}-\mathrm{F}$ bond weakened by hyperconjugation. Other band, at $1350 \mathrm{~cm}^{-1}$ can be ascribed to the stretching vibration of $-\mathrm{CF}_{2}$ moieties present in the defects/ edges formed by breaking of graphitic layers during fluorination. ${ }^{17}$ All those vibration bands disappear after exfoliation whatever the starting material, even for FCND.

To obtain more information on the structure of the sample FG-650, Raman spectra were measured (Fig. 5b), from which D/
$\mathrm{G}$ ratio were extracted in relation with the presence of defects and residual fluorine atoms on the surface. Moreover, both position and shape of the $2 \mathrm{D}$ band are analyzed to evidence the presence of graphene layers in the exfoliated sample and their stacking. At the same time, two new bands at 1615 and $2940 \mathrm{~cm}^{-1}$ can be observed in the Raman spectrum. Due to the high concentration of fluorine atoms, the too strong luminescence background avoided the recording at $514 \mathrm{~nm}$ for the starting materials. All the bands were fitted using simple Lorentzian, and the D/G ratio was 1.5, 1.7 and 1.1 for Exf-FG, Exf$F C N F$ and Exf-FCND, respectively, which is relatively high in comparison with reduced graphene. ${ }^{18}$ Both the presence of residual fluorine atoms and the increase of the amount of defects formed during the thermal exfoliation contribute to the high intensity of the D-band. $\mathrm{D}^{\prime}$ band, characteristic of few layer graphene sheets, appeared as a shoulder of the $\mathrm{G}$ band. ${ }^{\mathbf{1 8 , 1 9}}$ Concerning the $2 \mathrm{D}$ band, it may be a direct indicator for a graphene-like sheets with few layers. ${ }^{20}$ Here, exfoliation of the fluorinated graphite into fluorinated graphene was demonstrated from the change in position and shape of this band, which is sharp and symmetric.

Thanks to the presence of a high amount of fluorine atoms, the exfoliation of fluorinated graphite by a very fast thermal
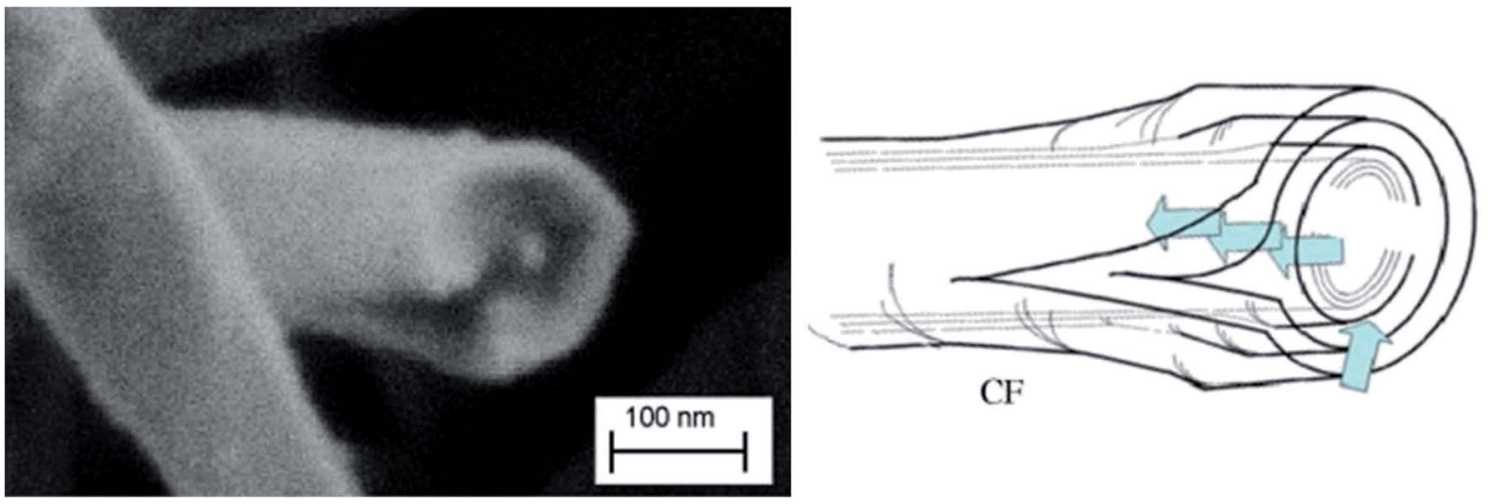

Fig. 3 SEM images and schematic view of FCNF. 

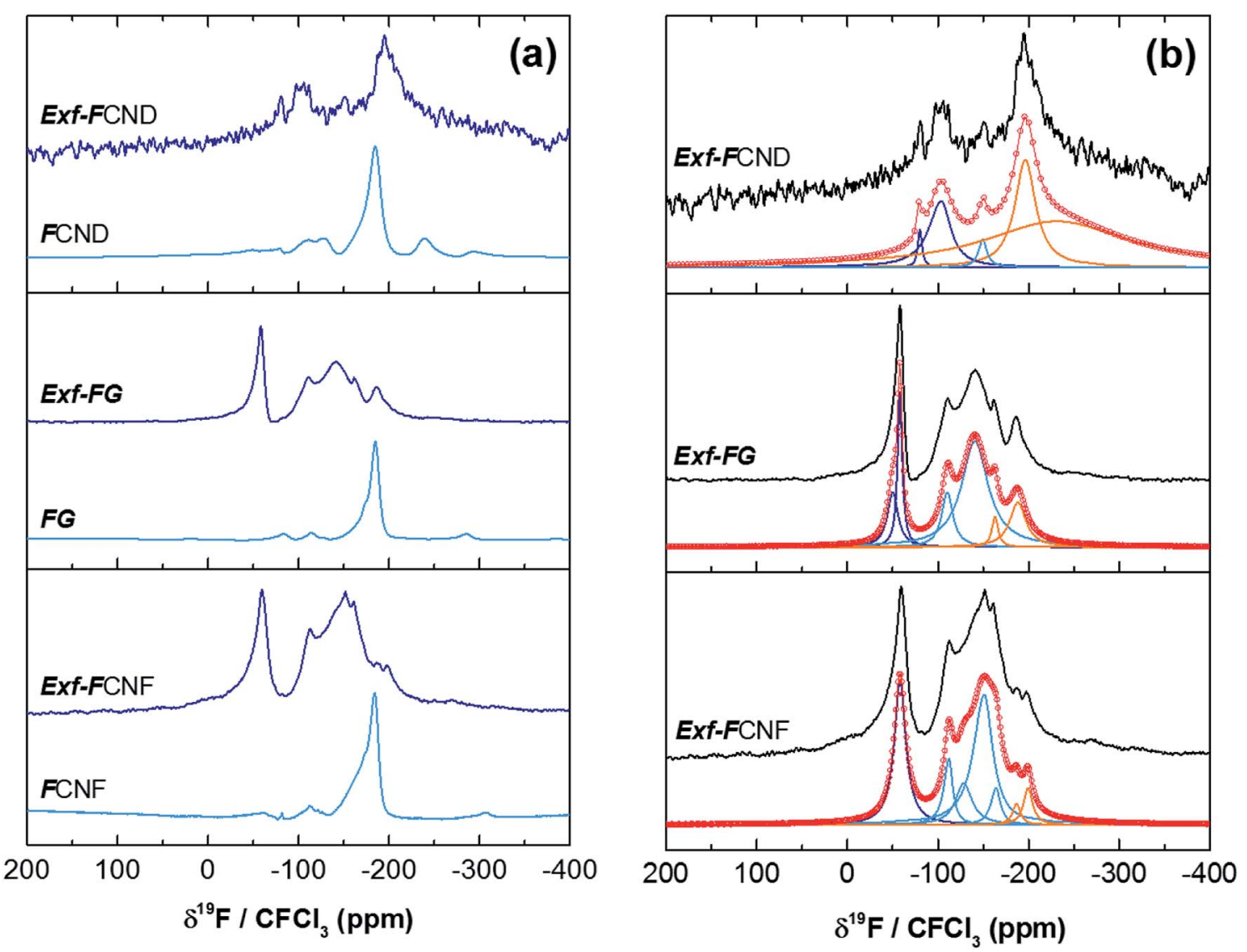

Fig. $4{ }^{19} \mathrm{~F}$ MAS spectra, at $34 \mathrm{kHz}$, of raw and exfoliated fluorinated carbons (a) and their fits (b).

Table 1 Relative content of the fluorinated groups $C F, \mathrm{CF}_{2}$ and $\mathrm{CF}_{3}$

\begin{tabular}{lccc}
\hline & Exf-FCND & Exf-GF & Exf-FCNF \\
\hline$\% \mathrm{CF}$ & 80.8 & 16.2 & 6.8 \\
$\% \mathrm{CF}_{2}$ & 2.3 & 60.4 & 69.9 \\
$\% \mathrm{CF}_{3}$ & 16.9 & 23.4 & 23.3
\end{tabular}

treatment has proved to be efficient for the case of graphite and nanofibers. The presence of fluorine favors the exfoliation, thanks to the generation of fluorinated gaseous species such as $\mathrm{CF}_{4}$ and $\mathrm{C}_{2} \mathrm{~F}_{6}$, which occurs simultaneously with the defluorination. When the treatment is performed with a fast temperature increase, both exfoliation and restructuring of the graphitic regions occur simultaneously during the flash. During the thermal shock, the $\mathrm{C}-\mathrm{F}$ bonding becomes weakened because of the fluorine atoms dilution in the sheets (most of $\mathrm{F}$ atoms are located in the sheet edges and defects in the forms of $\mathrm{CF}_{2}$ and $\mathrm{CF}_{3}$ groups). Highly fluorinated graphite or nanofibers appears as a promising precursor for the preparation of conductive graphene with low fluorine content. Contrary to graphene oxide, the reduction step to obtain pure graphene could be easier because of the low amount of residual fluorinated groups.

The exfoliation and gas evolution are hindered for the case of fluorinated nanodiscs. The accommodation of fluorine atoms results in small cracks homogenously dispersed on the disc surface. $^{21}$ During the thermal shock, fluorinated gases are removed both from those cracks and from edges of discs with average diameter of $1.5 \mu \mathrm{m}$; the resulting pressure between the carbon sheets is not enough to ensure huge exfoliation as for fluorinated graphite and nanofibers. Thermal exfoliation of nanodiscs was then not successful and the resulting sample appear close to the precursors for which the tribological properties were studied. ${ }^{\mathbf{8 - 1 1}}$ Tribological tests were then performed only for exfoliated nanofibers and graphite. Both the exfoliated structure and presence of residual fluorine may act on the friction energy.

\subsection{Friction properties}

The intrinsic friction coefficients, recorded at 10 cycles, of Exf$F G$ and $E x f-F C N F$ are presented in Fig. 6 in comparison with raw fluorinated materials. Fig. 6 shows that the exfoliation process does not influence the intrinsic friction performances of the compounds as $\mu=0.085 \pm 0.003$ and $\mu=0.083 \pm 0.002$ for $F G$ and $\operatorname{Exf}-F G$, respectively. In the case of carbon nanofibers, the intrinsic friction coefficients are lower, as $\mu=0.076 \pm 0.001$ for FCNF compared to $\mu=0.077 \pm 0.001$ for Exf-FCNF. For such compounds, we showed in previous works that the friction reduction mechanisms are associated to a lowering of interparticles interactions due to the fluorination of external graphene layers. ${ }^{8-10}$ It has then to be noticed that the observed 

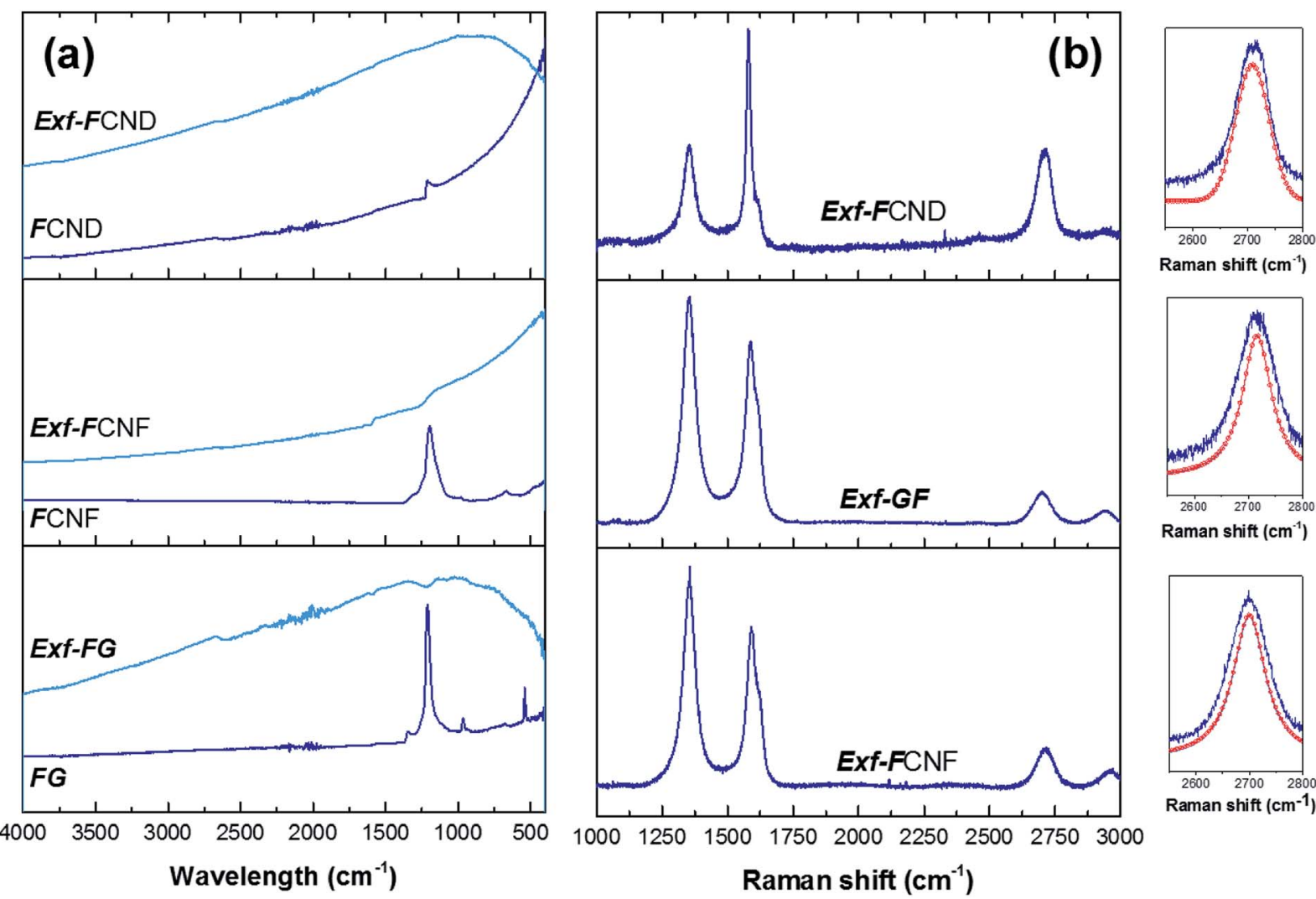

Raman shift $\left(\mathrm{cm}^{-1}\right)$

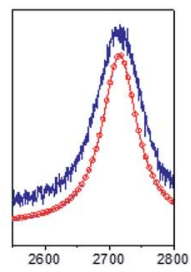

Raman shift $\left(\mathrm{cm}^{-1}\right)$

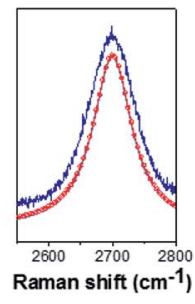

Fig. 5 FTIR (a) and Raman spectra (b) of raw and exfoliated. Insets display the fit of 2D band.

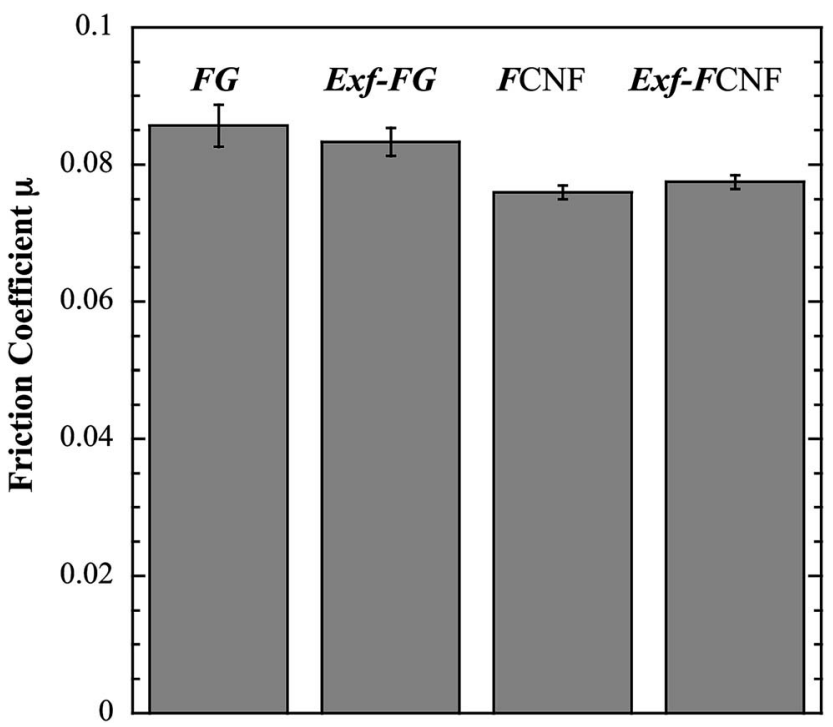

Fig. 6 Intrinsic friction coefficient, recorded at 10 cycles, of FG, ExfFG, FCNF and Exf-FCNF.

defluorination of the compounds during exfoliation does not deteriorate the lubricating performances of the tested materials. If considering the stability of $\mu$ for raw and exfoliated materials and the weak fluorination rate of Exf-FG and Exf$F \mathrm{CNF}$, the good friction properties seem to be related to the weakening of the interparticles interactions due to the exfoliation process rather than to the fluorine content. This is confirmed if we compare the friction coefficients of Exf-FCNF ( $\mu$ $=0.076$ ) and of fluorinated carbons with nearly the same fluorine content. The mild fluorination of graphitized carbon blacks and carbon nanofibers allowed $\mathrm{F} / \mathrm{C}$ atomic ratio of 0.08 and 0.05 , respectively, to be achieved. ${ }^{8-11}$ In comparison with raw nanocarbons, the decrease of the friction coefficient was then recorded for both CNFs and GCBs $(0.09 \pm 0.01$ and $0.11 \pm 0.01$, respectively); for the cases of raw GCBs $(0.12 \pm 0.01)$ and CNFs $(0.14 \pm 0.01)$, the friction coefficients are higher and close to those obtained for graphite in air (0.10-0.15).

The evolution of the friction coefficients of raw and exfoliated materials are reported in Fig. 7 in the case of graphite and Fig. 8 in the case of carbon nanofibers, as a function of cycle number. In the case of $F G$ and FCNF, Fig. 7 and 8 show an increase of the friction coefficient during the tribological tests. The friction coefficient becomes superior to 0.1 after 50 cycles for $F G$ and 100 cycles for $F C N F$. This is attributed to a tribofilm breakdown. On the contrary, the good lubricating performances of $E x f-F G$ and $E x f-F C N F$ are maintained after 1000 cycles of friction. Both peeling of some fluorinated graphene layers and decrease of the interparticle interaction could occur during the formation of the tribofilm as for the case of nanofibers and graphitized carbon blacks. ${ }^{8-10}$ An orientation of the fibers occurred during the formation of the tribofilm. ${ }^{8,9}$ In the same way, fluorinated GCBs could disaggregate and easily form 


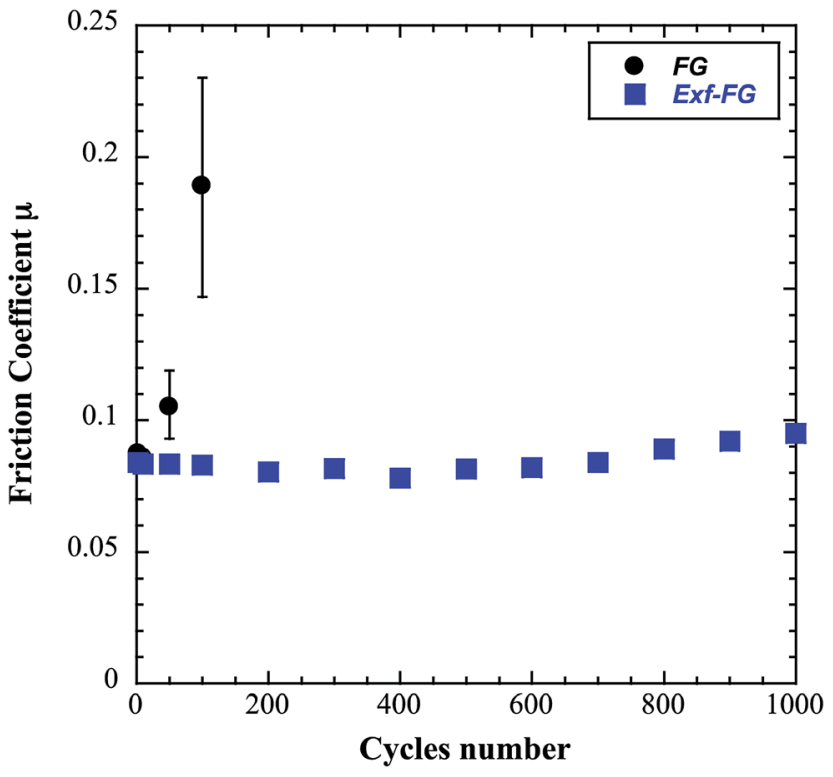

Fig. 7 Evolution of the friction coefficient as a function of cycles number in the case of $F G$ and Exf-FG.

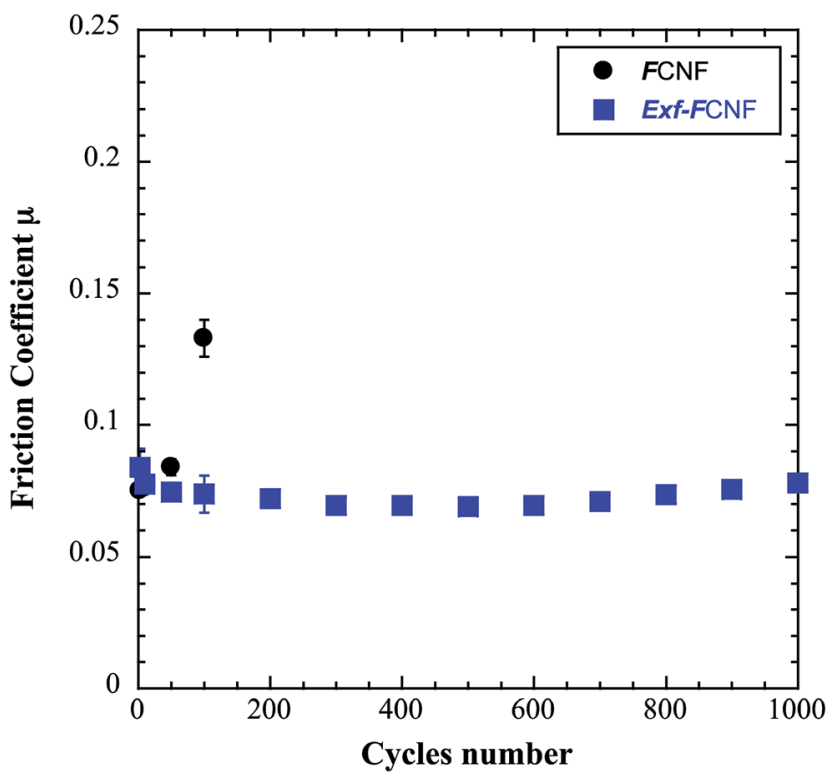

Fig. 8 Evolution of the friction coefficient as a function of cycle number in the case of FCNF and Exf-FCNF.

a dense tribofilm. The thickness of the film is of $0.6 \mu \mathrm{m} .^{8,9}$ The friction properties differ for GCBs and CNFs probably according to the nature of the tribofilm and suggest different friction mechanisms involving surface effects for the case of CNFs and bulk effects for the case of GCBs. For the case of exfoliated fluorinated graphite, the changes of friction coefficient suggest that the exfoliation process facilitates the formation of an homogeneous and stable tribofilm. Exfoliated particles enter more easily in the convergent zone of the contact and resulting in a deposition of graphene layers parallel to the sliding direction.

\section{Conclusion}

Thermal exfoliation of fluorinated carbons using a thermal shock is an efficient route to prepare solid with low friction coefficient as soon as the first friction cycle. In order to highlight the advantages of exfoliated fluorinated carbons as solid lubricants or as additives for lubricating oils, further work must be performed, i.e. wear rate, morphology of wear track and transferred film on the counter ball would be performed. The method may be used for numerous fluorinated precursors, e.g. high temperature graphite fluorides and fluorinated carbon nanofibers, if the gas evolved during the exfoliation can be removed through an opened (graphite fluorides) or defected structure (nanofibers). The case of fluorinated carbon nanodiscs evidences the opposite behavior where numerous regions (cracks and disc edges) allow the gases to be evolved that limits the pressure between carbon sheets and then the exfoliation. The thermal exfoliation is efficient whatever the $\mathrm{C}-\mathrm{F}$ bonding even for covalent character. Exfoliation and defluorination occur simultaneously resulting in samples with low fluorine content, with composition close to $\mathrm{CF}_{0.05}$. Although the massive defluorination, the comparison of raw and exfoliated fluorinated carbons evidences that exfoliation does not deteriorate the excellent lubricating performances because of the weakening of the interparticles interactions due to the exfoliation process. Exfoliated structure may facilitate the formation of an homogeneous and stable tribofilm, as for other fluorinated carbons, even if the low fluorine content is low. This constitutes the main difference with the corresponding fluorinated carbons with similar fluorine content.

\section{Conflicts of interest}

There are no conflicts to declare.

\section{References}

1 D. Berman, S. A. Deshmukh, S. K. R. S. Sankaranarayanan, A. E. Anirudha and V. Sumant, Extraordinary macroscale wear resistance of one atom thick graphene layer, $A d v$. Funct. Mater., 2014, 24(42), 6640-6646.

2 D. Berman, A. Erdemir and A. V. Sumant, Graphene: a new emerging lubricant, Mater. Today, 2014, 17(1), 31-42.

3 J. Taha-Tijerina, Multifunctional nanofluids with 2D nanosheets for thermal and tribological management, Wear, 2013, 302(1-2), 1241-1248.

4 V. Eswaraiah, V. Sankaranarayanan and S. Ramaprabhu, Graphene-based engine oil nanofluids for tribological applications, ACS Appl. Mater. Interfaces, 2011, 3(11), 42214227.

5 L.-F. Wang, T.-B. Ma, Y.-Z. Hu and H. Wang, Atomic-scale friction in graphene oxide: an interfacial interaction perspective from first-principles calculations, Phys. Rev. B: Condens. Matter Mater. Phys., 2012, 86, 125436.

6 J. Wang, T. Ma, Y. Hu, Q. Zheng, H. Wang and J. Luo, Theoretical study of superlow friction between two single- 
side hydrogenated graphene sheets, Tribol. Lett., 2012, 48, 255-261.

7 M. Dubois, K. Guérin, J. Giraudet, J. F. Pilichowski, P. Thomas, K. Delbé, J.-L. Mansot and A. Hamwi, Direct fluorination of poly(p-phenylene), Polymer, 2005, 46(18), 6736-6745.

8 P. Thomas, P. Bilas, A. Molza, L. Legras, J.-L. Mansot, K. Guerin and M. Dubois, Fluorinated Nanocarbons for Lubrication, in New Fluorinated Carbons: Fundamentals and Applications, ed. O. Boltalina, T. Nakajima and A. Tressaud, Elsevier Inc, 2016, ch. 14, pp. 325-360, ISBN 9780128034798.

9 N. Nomède-Martyr, E. Disa, P. Thomas, L. Romana, J.-L. Mansot, M. Dubois, K. Guérin, W. Zhang and A. Hamwi, Tribological properties of fluorinated nanocarbons with different shape factors, J. Fluorine Chem., 2012, 144, 10-16.

10 P. Thomas, D. Himmel, J. L. Mansot, W. Zhang, M. Dubois, K. Guérin and A. Hamwi, Friction properties of fluorinated carbon nanodiscs and nanocones, Tribol. Lett., 2011, 41(2), 353-362.

11 P. Thomas, J. L. Mansot, A. Molza, F. Begarin, M. Dubois and K. Guérin, Friction properties of fluorinated graphitized carbon blacks, Tribol. Lett., 2014, 56, 259-271.

12 S. N. Naess, A. Elgsaeter, G. Helgesen and K. D. Knudsen, Carbon nanocones: wall structure and morphology, Sci. Technol. Adv. Mater., 2009, 10, 065002.

13 S. Lynum, J. Hugdhal, K. Hox, R. Hildrum and M. Nordvik, Production of micro domain particles by use of a plasma process, Patent EP1017622, 2000.

14 (a) J. Giraudet, M. Dubois, K. Guérin, J. Pinheiro, A. Hamwi, W. Stone, P. Pirotte and F. Masin, J. Solid State Chem., 2005, 178, 1262-1268; (b) J. Giraudet, M. Dubois, A. Hamwi, W. E. Stone, P. Pirotte and F. Masin, J. Phys. Chem., 2005,
109, 175-181; (c) J. Giraudet, C. Delabarre, K. Guérin, M. Dubois, F. Masin and A. Hamwi, J. Power Sources, 2006, 158, 1365-1372; (d) J. Giraudet, M. Dubois, K. Guérin, C. Delabarre, A. Hamwi and F. Masin, J. Phys. Chem. B, 2007, 111, 14143-14151.

15 M. Dubois, K. Guerin, Y. Ahmad, N. Batisse, M. Mar, L. Frezet, W. Hourani, J. L. Bubendorff, J. Parmentier, S. Hajjar-Garreau and L. Simon, Thermal exfoliation of fluorinated graphite, Carbon, 2014, 77, 688-704.

16 Y. Sato, K. Itoh, R. Hagiwara, T. Fukunaga and Y. Ito, On the so-called "semi-ionic" C-F bond character in fluorine-GIC, Carbon, 2004, 42, 3243-3249.

17 P. W. Gong, Z. F. Wang, J. Q. Wang, H. G. Wang, Z. P. Li, Z. J. Fan, Y. Xu, X. X. Han and S. R. Yang, One-pot sonochemical preparation of fluorographene and selective tuning of its fluorine coverage, J. Mater. Chem., 2012, 22, 16950-16956.

18 X. Diez-Betriu, S. Alvarez-Garcia, C. Botas, P. Alvarez, J. Sanchez-Marcos, C. Prieto, R. Menendez and A. de Andres, Raman spectroscopy for the study of reduction mechanisms and optimization of conductivity in graphene oxide thin films, J. Mater. Chem. C, 2013, 1, 6905-6912.

19 W. H. Lee, J. W. Suk, H. Chou, J. Lee, Y. Hao, Y. Wu, R. Piner, D. Akinwande, K. S. Kim and R. S. Ruoff, Selective-area fluorination of graphene with fluoropolymer and laser irradiation, Nano Lett., 2012, 12, 2374-2378.

20 A. C. Ferrari, Raman spectroscopy of graphene and graphite: disorder, electron-phonon coupling, doping and nonadiabatic effects, Solid State Commun., 2007, 143, 47-57.

21 W. Zhang, M. Dubois, K. Guérin, P. Bonnet, E. Petit, N. Delpuech, D. Albertini, F. Masin and A. Hamwi, Effect of graphitization on fluorination of carbon nanocones and nanodiscs, Carbon, 2009, 47(12), 2763-2775. 